\title{
Modelling rhythmic function in a musician post-stroke
}

\author{
Sarah J. Wilson ${ }^{\mathrm{a}, *}$, Jeffrey L. Pressing ${ }^{\mathrm{a}}$, Roger J. Wales ${ }^{\mathrm{b}}$ \\ ${ }^{a}$ Department of Psychology, School of Behavioural Science, The University of Melbourne, 3010, Victoria, Australia \\ ${ }^{\mathrm{b}}$ Faculty of Humanities and Social Sciences, La Trobe University, Bundoora, 3083, Victoria, Australia
}

Received 1 August 2001; accepted 6 September 2001

\begin{abstract}
The aim of this study was to model the components of rhythmic function in a case (H.J.) of acquired rhythmic disturbance. H.J. is a right-handed, amateur male musician who acquired arrhythmia in the context of a global amusia after sustaining a right temporoparietal infarct. His rhythmic disturbance was analysed in relation to three independent components using an autoregressive extension of Wing and Kristofferson's model of rhythmic timing. This revealed preserved error-correction and motor implementation capacities, but a gross disturbance of H.J.'s central timing system (“cognitive clock”). It rendered him unable to generate a steady pulse, prevented adequate discrimination and reproduction of novel metrical rhythms, and partly contributed to bi-manual co-ordination difficulties in his instrumental performance. The findings are considered in relation to the essential components of the cognitive architecture of rhythmic function, and their respective cerebral lateralisation and localisation. Overall, the data suggested that the functioning of the right temporal auditory cortex is fundamental to 'keeping the beat' in music. The approach is presented as a new paradigm for future neuropsychological research examining rhythmic disturbances. () 2002 Elsevier Science Ltd. All rights reserved.
\end{abstract}

Keywords: Rhythmic timing; Arrhythmia; Amusia; Music psychology

\section{Introduction}

A disturbance of rhythmic sense (arrhythmia) has been frequently reported in the amusia case literature. This literature examines the skills of musicians following acquired brain injury (for a review see $[2,60,61]$ ). A range of rhythmic deficits has been described, including the inability to reproduce rhythmic patterns or to discriminate between them, and the inability to perform music rhythmically, or to keep time to music, including dancing in time. These deficits may occur in combination with other musical deficits, such as impaired vocal and instrumental skills [2,3].

Despite the frequent co-occurrence of melodic and rhythmic impairments, systems responsible for their processing are thought to be relatively independent. Isolated deficits of 'amelodia' and arrhythmia have been reported in the amusia literature for perceptual, productive, and musical reading tasks $[4,13,30,37,38,40-42,62]$. Separate cognitive representations of melody and rhythm have also been proposed in the developmental $[62,63]$ and normal adult literature $[43,60,62]$, supporting the general applicability of this distinction.

\footnotetext{
* Corresponding author. Tel.: +61-3-8344-7391; fax: +61-3-9347-6618. E-mail address: s.wilson@psych.unimelb.edu.au (S.J. Wilson).
}

Taking this approach one step further, Peretz and co-workers $[25,37,43]$ argued that rhythmic and metric organisation can be differentially impaired in brain damaged patients, supporting the relative independence of functions of the rhythmic system. In terms of cerebral lateralisation, Peretz and Morais [43] suggested that metric organisation may be ascribed to the right hemisphere, whilst smaller rhythmic groupings are encoded by the left hemisphere. This notion is consistent with research findings from normal subjects [12,18,52] and brain-damaged patients [11,30,44], as well as data supporting left hemispheric specialisation of temporal processing relevant to speech perception $[33,34,51,54]$. Neuropsychological studies of groups of patients, however, have generally shown more variable lateralisation effects, particularly in recent research $[25,36,46]$. Moreover, as noted by Penhune et al. [36], temporal processing relevant to speech perception has been typically evaluated within a brief time frame $(2-50 \mathrm{~ms})$ in comparison to the temporal processing of musical rhythms (period of seconds), making it difficult to generalise across task effects [36].

Apart from the observed dissociation of rhythmic and metric organisation, few studies have examined the cognitive architecture of rhythmic function based on a detailed analysis of acquired rhythmic disturbance. The use of theoretical models of the functioning of the rhythmic system 
is generally lacking in the neuropsychological literature, and the neurological basis of arrhythmia has been subject to much speculation [17,39]. Exceptions include a case reported by Mavlov [30] of a 61-year old, right-handed, professional male musician (violinist and music teacher) who suffered a cerebrovascular accident in the left posterior parietal region. This musician displayed impaired rhythmic pattern discrimination and reproduction across auditory, visual and tactile stimuli. Mavlov characterised the impairment as a 'supramodal' rhythmic disorder, considered to underlie a severe amusia in this case.

More recently, Fries and Swihart [11] described the case of a 56-year-old, left-handed, amateur male musician who played the drums and the bugle. He presented with a unique disturbance of rhythmic function, in that he was able to correctly discriminate and produce rhythmic patterns, but he could no longer produce rhythmic behaviour "... in phase with an externally given acoustic rhythm source" (p.1317). He displayed a modality specific disturbance of auditorily paced predictive motor behaviour that affected activites such as walking, dancing, singing and speech. This occurred following infarction of the right temporal lobe with additional right basal ganglia damage. The rhythmic deficit was accompanied by sensory amusia and poor pitch and rhythmic production during spontaneous singing (vocal amusia).

The present study focuses on the case of H.J., a 67-year-old right-handed, amateur male musician who suffered a right temporoparietal infarct, accompanied by a severe global amusia [60,61]. This included arrhythmia, with paced and unpaced production both impaired. The aim of this paper was to provide a detailed analysis of H.J.'s rhythmic disturbance. A cognitive model of rhythmic timing derived from normal subjects in the experimental psychology field $[47-49,58,68,69]$ was employed to analyse the essential components of this disturbance.

\subsection{The Wing and Kristofferson model of rhythmic timing}

Research examining the timing of rhythmic production in normal subjects has typically employed a two-tiered cognitive model. This model was originally enunciated by Wing and Kristofferson $[68,69]$ and has received substantial empirical support across a range of experimental contexts [19,50,57,65-67]. For self-generated, isochronous tapping, the basic model comprises two independent components. The first is a "cognitive clock" that internally generates "timekeeper pulses" defining regular intervals $\left(C_{n}\right)$. This is considered to have a central source within the nervous system; in other words, the "cognitive clock" is equivalent to a "central time keeper". The second component involves a more peripheral motor response, each triggered by one timekeeper pulse. Each cognitive clock pulse starts a clock interval $\left(C_{n}\right)$ and initiates a motor response that can be characterised by a certain implementation time, or motor delay $\left(D_{n}\right)$. Given these two definitions, the intertap interval
$\left(I_{n}\right)$ between successive taps can be expressed by

$I_{n}=C_{n}+\left(D_{n+1}-D_{n}\right)$

Using Wing and Kristofferson's basic model [68,69], independent estimates of the motor delay and clock variances can be obtained. Various studies have provided support for the independence of these variables, including their dissociation in patients with Parkinson's disease or cerebellar damage $[19,20]$. The motor delay variance has been found to be relatively independent of tapping period, whilst clock variance has been shown to have an approximate quadratic relationship with it $[19,47,66]$.

Tapping in response to an externally generated pulse (paced tapping) can also be incorporated into the Wing and Kristofferson approach [49,58]. A fourth term must be added to Eq. (1), based on the asynchrony $\left(A_{n}\right)$ between a subject's tap and the externally generated signal (typically a recurring audio tone) at time $n$. The asynchrony is presumably utilised by a cognitive process that linearly adjusts for any differences between the externally generated pulse and the motor output. Thus, the extended model contains an error-correction term $\left(-\alpha A_{n}\right)$ that allows for correction of the clock interval $\left(C_{n}\right)$ according to the most recent asynchrony between tone and tap [58]

$I_{n}=C_{n}-\alpha A_{n}+\left(D_{n+1}-D_{n}\right)$

As shown in Eq. (2), the interresponse interval is defined in terms of the sum of the internal sources of variation plus the error-correction mechanism, which is linear. Importantly, error-correction is a third, independent process, completely autonomous from the clock and motor mechanisms. Alpha is the (first order) error-correction coefficient which is independent of the scale of the asynchronies. It indicates the rapidity and strength of error-compensation, which presumably relates directly to both information transmission and processing $[47,49]$. This extended model has been referred to as the AR1 model [49], since it is first order autoregressive. It has been considered by previous researchers in various forms (see $[16,28,29,53,58]$ ).

The use of paced tapping incorporates explicit errorcorrection. This was essential in assessing the nature of H.J.'s arrhythmia, as he was unable to reliably perform self-generated (unpaced) isochronous tapping. This is the first study known to the authors to examine the error-correction model in a brain damaged, arrhythmic musician.

Fig. 1 provides a diagrammatic representation of the Wing and Kristofferson model, extended by Vorberg and Wing [58] to incorporate the asynchrony variable associated with an external tone source. As can be seen from this figure, the model comprises three tiers, whereby the cognitive clock and motor systems must become closely entrained to the externally generated reference tone. When asynchrony is non-zero, adjustment is made to the motor output via the cognitive clock system. 


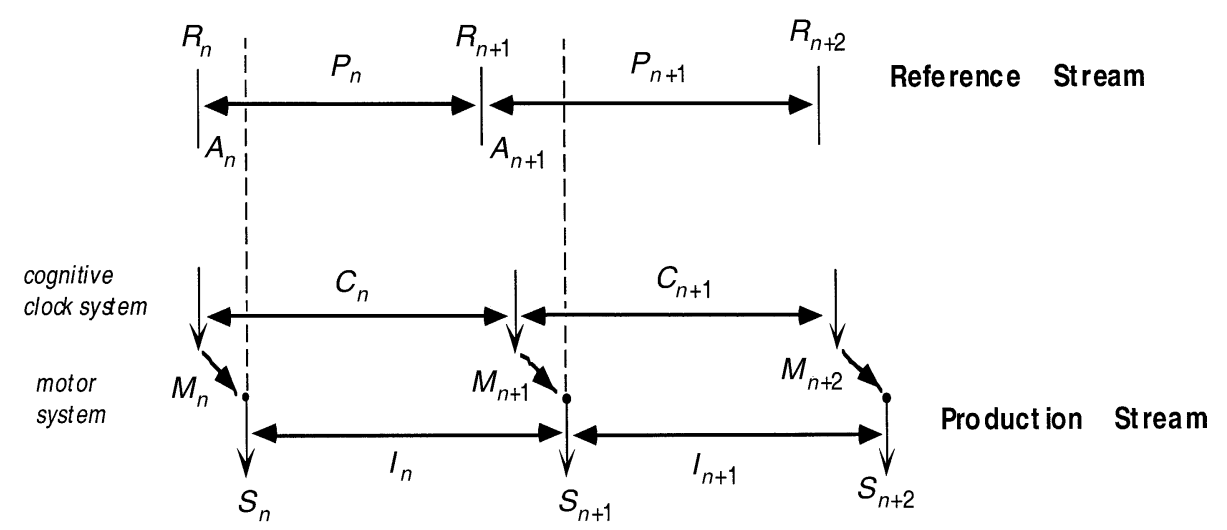

Fig. 1. The Vorberg and Wing synchronisation approach showing the co-ordination of tapping production with regular audio tones [58]. $\left(P_{n}=\right.$ interval between the synchronising tones; $C_{n}=$ cognitive clock intervals; $M_{n}=$ motor delays; $S_{n}=$ subject taps; $I_{n}=$ interresponse intervals).

A further development of the model instigated by Pressing and Jolley-Rogers [49] involved the inclusion of two-error terms, with designated error parameters $\alpha$ and $\beta$. The inclusion of two such terms allows for the condition in which a subject may base error-correction on the asynchrony of the tone at time $n$ as well as the tone previous to that, $n-1$. This model (designated as AR2-second order autoregressive) represents a more complex or sophisticated timing system than that posed by AR1. Its form is

$I_{n}=C_{n}-\alpha A_{n}-\beta A_{n-1}+\left(D_{n+1}-D_{n}\right)$

As evident from Eqs. (2) and (3), the AR1 model is a subcase of AR2 ( $\beta=0$ yields AR1), since AR2 has two free error-correction parameters ( $\alpha$ and $\beta$ ), and AR1 only one $(\alpha)$. AR2 provides, therefore, a less parsimonious account of rhythmic timing in response to an externally generated tone. Its use is only mandated when experimental data show an inferior fit to the AR1 model.

The recent study by Pressing and Jolley-Rogers [49] revealed that the AR2 model was better suited for simple tapping data derived from a 48-year-old, expert musician performing the tapping task at very fast speeds $(\leq 150 \mathrm{~ms}$ or 20 taps in every $3 \mathrm{~s}$ ). For slower speeds, and for a non-musician, the simpler AR1 model was consistently preferred. Pressing and Jolley-Rogers interpreted these findings in relation to an auditory reaction time zone $(150-200 \mathrm{~ms})$, where error-correction begins to decline due to auditory processing limitations. In the case of the musician, this may be overcome by the use of an 'expert system', represented by the AR2 model. Other conditions under which such advanced expertise appeared are described in Pressing [48].

H.J.'s performance of the tapping task was considered in relation to the extended error-correction model. Eq. (3) can be solved for $\alpha, \beta$, and the variances of the clock and motor processes, using the mathematical procedures outlined by Pressing and Jolley-Rogers [47,49]. This enabled H.J.'s rhythmic timing difficulties to be quantified in terms of the functioning of his cognitive clock, motor system, and error-correction processes. The results were considered in relation to both the AR1 and AR2 cases to determine which model best characterised his performance.

Given that the details of the theoretical issues underpinning the models are not addressed here, the interested reader is referred to the indicated original references for a thorough account of these issues. For the purposes of this paper, however, we believe it is useful to list the basic assumptions underlying our models.

1. The process controlling the synchronisation task is stationary over the course of a run.

2. "Clock" (central) and "motor" (peripheral) sources of noise are independent.

3. Error-correction is based linearly on asynchrony and is achieved by local correction of the central clock process.

\section{Methods}

An in-depth assessment of H.J.'s musical skills was conducted between April, 1994 and February 1996, as part of a larger research program [60]. The results of this assessment have been previously reported, including a detailed account of H.J.'s case history, his neurological status, and neuropsychological functioning [61]. A summary of his details relevant to the present experiment will be provided here.

\subsection{Subjects}

At the commencement of testing (1994), H.J. was a 67-year-old, right-handed male musician. Six months previously, he had suffered a right, temporoparietal infarct, thought to have arisen as a complication of coronary angiography. A computed tomography (CT) scan of H.J.'s brain 3-months post-stroke revealed an ischaemic event in the territory of the inferior division of the right middle cerebral artery. It primarily involved auditory association cortex of the middle temporal gyrus and the posterior portion of the superior temporal gyrus, representing the right-sided equivalent of Wernicke's area. It extended back into the 


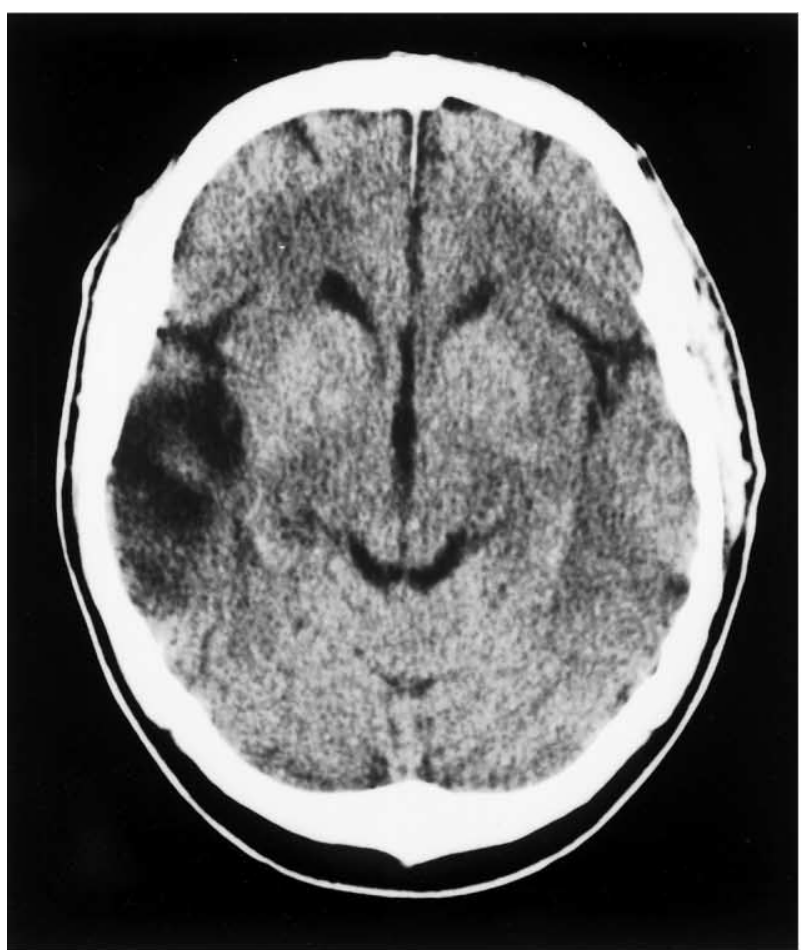

(a)

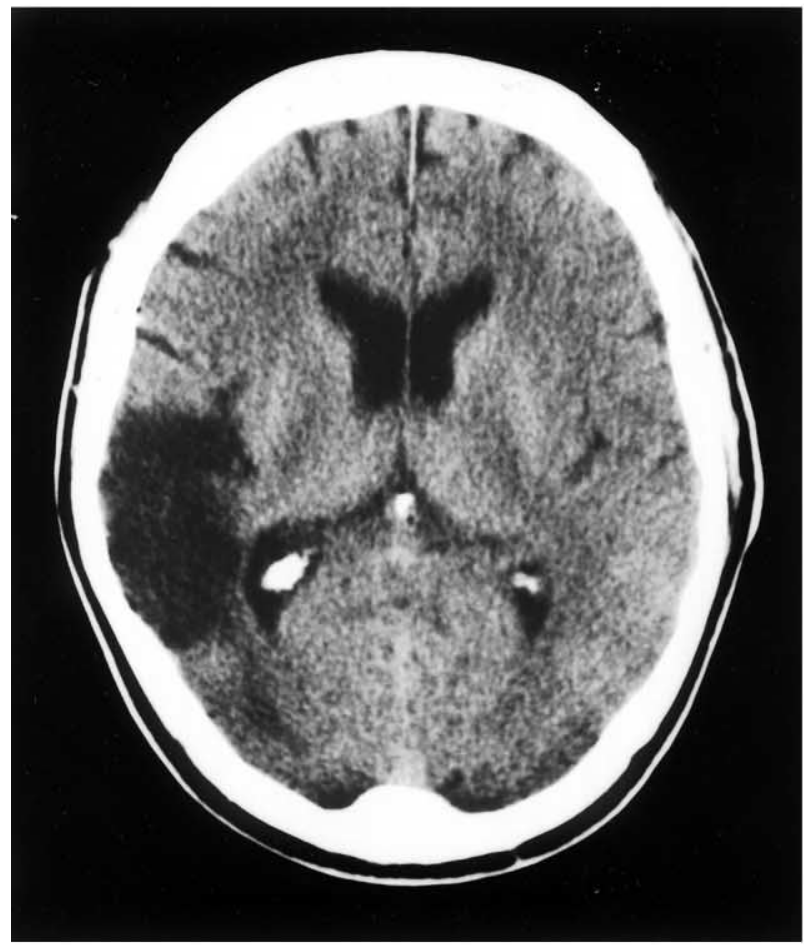

(b)

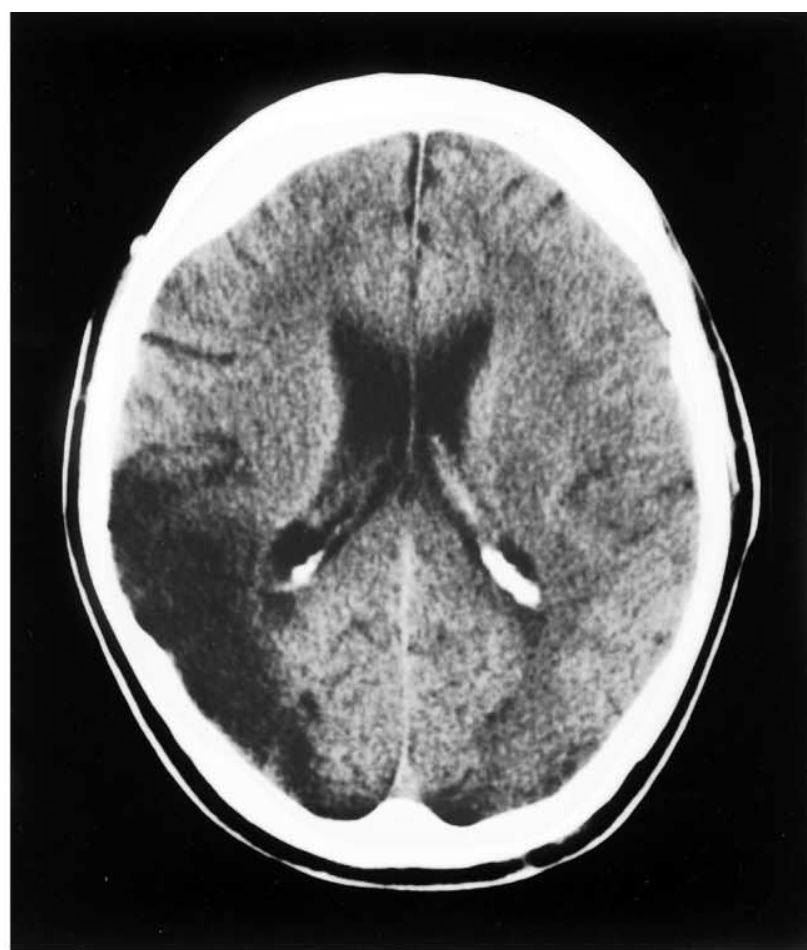

(c)

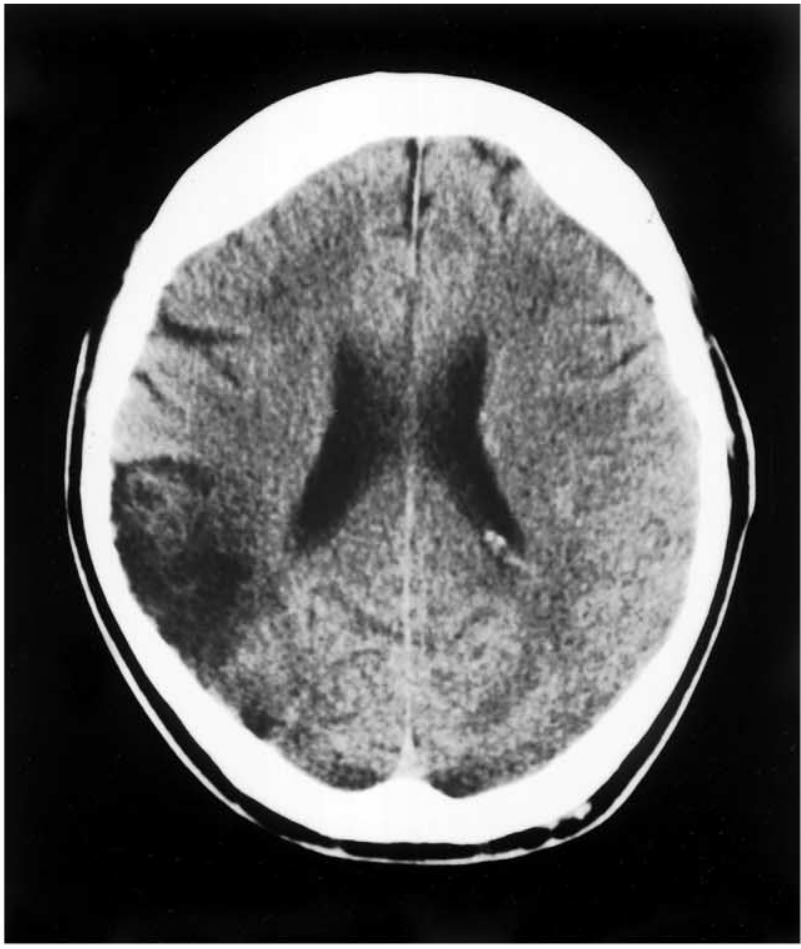

(d)

Fig. 2. Representative axial slices from H.J.'s CT scan, 3 months post-stroke [61]. The right hemisphere appears on the left-hand side of the scan, with the sequence of slices moving from lower to higher levels within the brain. The scan shows an ischaemic event in the territory of the inferior division of the right middle cerebral artery, involving auditory association cortex of the middle temporal gyrus and the posterior portion of the superior temporal gyrus, extending back into the angular gyrus and terminating at the temporo-parieto-occipital junction. 
angular gyrus of the inferior parietal lobule, and terminated at the temporo-parieto-occipital junction (Fig. 2). H.J. had a longstanding history of ischaemic heart disease, but no previous history of cerebral insult or psychiatric disorder.

H.J. commenced musical training at the age of 6 years, acquiring skills on a range of instruments including the piano, the piano accordian, the electric theatre organ, the guitar, the clarinet, the drums and the harmonica, although his principal instrument was the piano. He was also an accomplished tenor singer. Following his stroke, H.J. complained of a number of musical difficulties, including an inability to "comprehend" music when listening or performing, difficulty "transferring music from his head into his hands" when wanting to play, and difficulty dancing in time to music.

H.J. had 10 years of formal education, and a full-scale IQ (FSIQ) of 105 as measured on the Wechsler adult intelligence scale-revised [59]. Repeated neuropsychological assessments following his stroke revealed impaired visuoperceptual and spatial functions, as well as a mild constructional apraxia. Initially, there was evidence of left hemispatial neglect, which had largely resolved 6-months post-stroke. There was no evidence of difficulties on tasks of psychomotor speed, facial or ideomotor apraxia, language, memory, or frontal lobe functions $[60,61]$.

Neurobehavioural assessment of H.J. post-stroke showed reduced tactile sensation on the left, with midline sensory extinction. There was also evidence of partial dysgraphaesthesia and imprecise movements of the left hand. Motor functioning of H.J.'s upper and lower limbs was tested using Denckla's battery of motor co-ordination tasks [7], compared to the mean performance of a group of 10-year-old boys [7], and six adult control subjects (see below). Overall, H.J. showed superior performance of the motor tasks, as indicated by his faster speeds and normal power (Table 1). Qualitative observations, however, revealed clumsy and arrhythmical left-sided movements, which were felt to have a sensory rather than a pure motor basis $[60,61]$. Audiological assessment of H.J. post-stroke showed mild bilateral hearing loss in the high frequency range. This was considered typical of normal ageing (presbycusis) [60,61].

A normal control (NC) group of six male musicians was selected to match the musical background and premorbid musical ability of H.J as closely as possible. They had a mean age of 67 years (range 62-70 years), a mean education of 11.7 years (range 7.0-21.0 years), and a mean FSIQ of 114.2 (range 96.0-125.0 years). In comparison to the NC group, H.J. displayed a range of receptive musical difficulties post-stroke $[60,61]$. These included impaired discrimination of musical pitch, chords and melodic patterns, a disturbance of pitch working memory, a loss of tonal knowledge (atonalia), and difficulty recognising familiar tunes not associated with lyrical content (Table 2). These impairments were not attributable to a language or general auditory disurbance (such as nonverbal auditory agnosia), but rather, were specific to musical stimuli $[2,17,38,39,71]$.

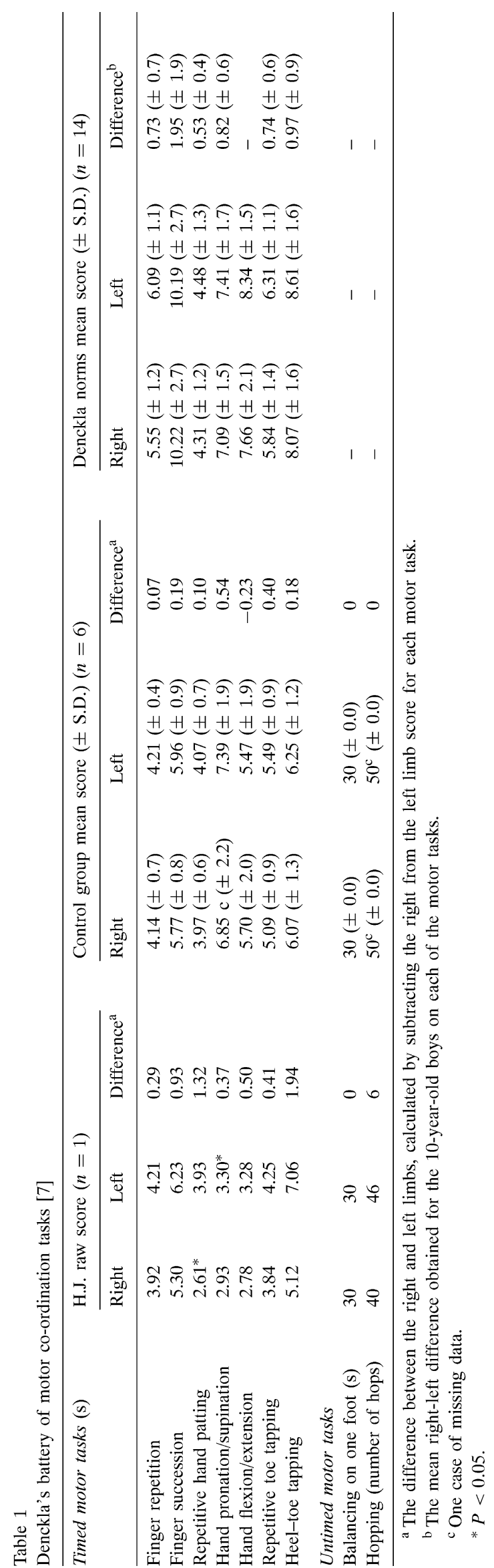


Table 2

Summary of H.J.'s musical skills [60,61]

\begin{tabular}{|c|c|c|c|c|c|}
\hline $\begin{array}{l}\text { H.J.'s pitch and rhythmic skills } \\
\text { (compared to the NC group) }\end{array}$ & Normal & Impaired & $\begin{array}{l}\text { H.J.'s vocal and instrumental skills } \\
\text { (compared to premorbid performance) }\end{array}$ & Normal & Impaired \\
\hline Pitch discrimination & & & Vocal Performance & & \\
\hline Sine tones $[1]$ & $\checkmark$ & & Pitch reproduction & & $\checkmark$ \\
\hline Piano tones & & $\checkmark^{* * *}$ & Novel melody reproduction & & $\checkmark$ \\
\hline Chord discrimination $[1]$ & & $\checkmark^{* *}$ & Production of a familiar tune & $\checkmark^{\mathrm{a}}$ & \\
\hline Melodic discrimination (5-note melody) & & & Production of tonal endings & & $\checkmark$ \\
\hline 3 notes changed $[64]$ & $\checkmark$ & & Instrumental Performance-Piano & & \\
\hline 1 note changed [1] & & $\checkmark^{* * *}$ & (i) Familiar pieces & & \\
\hline Pitch working memory $[71]^{\mathrm{b}}$ & & & Technique (finger patterns, dexterity) & & $\checkmark$ \\
\hline Short-term delay $(1650 \mathrm{~ms})$ & & $\checkmark^{* * *}$ & Bi-manual co-ordination & & $\checkmark$ \\
\hline Intermediate-term delay $(4000 \mathrm{~ms})$ & $\checkmark^{\mathrm{c}}$ & & Melodic/harmonic content & & $\checkmark$ \\
\hline Familiar tune identification & & & Rhythmic content & & $\checkmark$ \\
\hline $\begin{array}{l}\text { Not associated with lyrical content } \\
\text { Choruses played without lyrics }\end{array}$ & $\checkmark$ & $\checkmark^{* *}$ & $\begin{array}{l}\text { Use of dynamics/phrasing } \\
\text { (ii) Sight-reading }\end{array}$ & & $\checkmark$ \\
\hline Tonal identification & & & Right-hand passages & $\checkmark$ & \\
\hline Major/minor classification ${ }^{\mathrm{e}}$ & & $\checkmark^{* * *}$ & Left-hand passages & & $\checkmark$ \\
\hline Tonal/atonal classification [64] & & $\checkmark^{* * *}$ & Other Instrumental Performances & & \\
\hline Sound Identification & & & Organ & & $\checkmark^{\mathrm{f}}$ \\
\hline Musical timbres & $\checkmark$ & & Piano accordian & & $\checkmark^{\mathrm{f}}$ \\
\hline Environmental sounds & $\checkmark$ & & $\begin{array}{l}\text { Clarinet } \\
\text { Harmonica }\end{array}$ & & $\begin{array}{l}\checkmark^{\mathrm{g}} \\
\checkmark^{\mathrm{g}}\end{array}$ \\
\hline $\begin{array}{l}\text { Rhythmic timing } \\
\text { Clapping in time with simple music }\end{array}$ & & $\checkmark$ & Simultaneous vocal and instrumental performance & & $\checkmark$ \\
\hline Rhythmic discrimination & & & & & \\
\hline 5-Note rhythms; 3 or 4 notes changed [64] & & $\checkmark^{* *}$ & & & \\
\hline Metrical rhythms & & $\checkmark^{* * *}$ & & & \\
\hline Non-metrical rhythms & $\checkmark$ & & & & \\
\hline 4-Beat metrical rhythms; 1 beat changed [1] & & $\checkmark^{* * *}$ & & & \\
\hline Metrical/non-metrical classification [64] & $\checkmark$ & & & & \\
\hline Rhythmic pattern reproduction $^{\mathrm{h}}$ & & $\checkmark^{* * *}$ & & & \\
\hline Production of a familiar rhythm & $\checkmark$ & & & & \\
\hline
\end{tabular}

${ }^{a}$ Despite H.J.'s high level of pitch and rhythmic accuracy, his voice quality was characterised by a dull flattened tone that lacked musical expression.

${ }^{\mathrm{b}}$ These memory tests were developed by Dr. Robert Zatorre and used with his kind permission.

${ }^{\mathrm{c}}$ There was a trend towards impairment on this task.

${ }^{\mathrm{d}}$ H.J. was able to correctly name the notes in both the treble and bass clefs for these simple passages of one-handed sight-reading.

${ }^{\mathrm{e}}$ One control subject was excluded from this task.

${ }^{\mathrm{f}}$ H.J.'s performance of these instruments was characterised by difficulties identical to those listed for the piano. For the organ, H.J. also showed impaired use of the foot pedals, whilst use of the bellows of the piano accordian was characterised by poor bi-manual co-ordination and impaired rhythmic timing.

${ }^{\mathrm{g}}$ H.J. refused to play these instruments stating that he no longer remembered how to play even the most elementary songs.

${ }^{\mathrm{h}}$ Patterns were presented with or without accompanying pitch.

** $P<0.01$.

${ }^{* * *} P<0.001$.

H.J.'s musical performance skills were examined by obtaining repeated audio and visual recordings of his vocal and instrumental output. These were assessed relative to productions acceptable for public performance, given H.J.'s premorbid performance history. The recordings showed clear evidence of stable, vocal and instrumental difficulties, including incorrect pitching of tones and short novel melodies, and deficient intonation in the production of familiar tunes (Table 2). His instrumental performance was characterised by a lack of musicality, as well as the inability to produce an error free or continuous musical production. Errors were associated with a loss of appropriate finger patterns and occurred bilaterally, despite being more prominent in the left hand. They were accompanied by impaired bi-manual co-ordination, including difficulties integrating the timing of the two hands. These problems were observed for all musical instruments tested, and were most evident for the performance of recently learnt material (prior to the stroke), compared with highly familiar, over-learned tunes (Table 2).

In an attempt to assist H.J. with his instrumental performance, he was asked to play in time with an external pulse. This revealed that he was only able to 'keep the beat' for brief periods (less than $10 \mathrm{~s}$ ), after which he would lose all sense of pulse in his performance. In contrast to the $\mathrm{NC}$ group, he was also unable to clap in time with simple musical pieces, and showed impaired discrimination and reproduction of novel metrical rhythms (Table 2). The discrimination of non-metrical rhythms and their classification relative to metrical rhythms was less affected. These difficulties pointed towards an underlying disturbance of rhythmic timing. 


\subsection{Procedure}

H.J. performed the paced synchronisation task under laboratory conditions, according to the procedure outlined by Pressing and Jolley-Rogers [49]. He was seated in a soundproof booth, wearing headphones through which a repeating auditory tone was received bilaterally. The repeating tone had a frequency of $1000 \mathrm{~Hz}$, a duration of $30 \mathrm{~ms}$, and was presented at a comfortable listening volume, as determined by H.J. In response to the tone, he was asked to tap one of two force-sensitive keys (Honeywell microswitch PK89133) placed before him on a flat table, using first the right and then the left hand in alternate runs. The keys were connected to an IBM 486 computer equipped with special recording and data analysis software, as previously described by Pressing and co-workers [47-50]. The measurement accuracy of the equipment was between 1-2 ms.

H.J. performed the tapping task in response to five different speeds. The first was considered to represent a slow speed $($ period $=1000 \mathrm{~ms})$, requiring one tap per second. The last was a fast tempo (period $=200 \mathrm{~ms}$ ) considered capable of challenging an expert musician, requiring five taps per second. Intermediate speeds involved a gradual increase in tapping speed between this range (periods of 650,450 and $300 \mathrm{~ms}$ respectively).

Each experimental condition was administered a minimum of four times, separately for the two hands, to ensure that a large sample of taps was obtained. The speed of the runs was presented in counterbalanced order, alternating between the use of the right and left hands. Breaks were provided throughout the experiment to minimise errors resulting from decreased concentration and fatigue. Despite this, H.J. was unable to perform all of the conditions of the tapping task on the same day. Thus, administration of the faster speeds (periods of $\leq 450 \mathrm{~ms}$ ) was presented to H.J. on a separate testing occasion.

H.J.'s performance was compared to the performance of two normal control, male musicians, who performed the tapping task under similar conditions. One control was drawn from the $\mathrm{NC}$ group of six male musicians previously described (control 1), whilst the other was a new subject (control 2), again selected to match the age, musical background and premorbid musical ability of H.J. as closely as possible.

\subsection{Data analysis}

The 'binned' analysis technique was used to analyse the tapping data (see Pressing [47] for details). Exploratory data analysis resulted in exclusion of some of the data for each subject, particularly, at the onset of a run whilst they became accustomed to the pulse of the auditory tone. As a general rule, values greater than four standard deviations from the mean of a given run were also excluded as outliers, unless there was a compelling reason to suggest that such data were not aberrant. These data conditioning and exclusion criteria are standard to the research field, and provide a more stable estimation of the subjects' tapping performance.

Unlike the control subjects, H.J. was unable to track the fastest tapping speed $(200 \mathrm{~ms})$ in either the right or the left hand, showing complete breakdown of his rhythmic production at this challenging speed. In general, all comparable data were similar for the left and right hands of H.J. and the control subjects. Thus for the purpose of this study, H.J.'s performance was compared to the mean performance of the control subjects using the binomial sign test [5], collapsed across hands for each condition.

\section{Results}

\subsection{The extended Wing and Kristofferson model}

\subsubsection{Cognitive clock pulses $\left(C_{n}\right)$}

As shown in Fig. 3, the most striking effects were observed for H.J.'s cognitive clock. In comparison to the control subjects he displayed significantly greater variance across the conditions of the tapping task (binomial sign test, $P=0.004)$. This gross disturbance likely reflects a lesion-induced deficit.

The data from all subjects were well fit by a linear increase in clock variance as a function of period (1/tapping speed; see Fig. 3). This is typical of the performance of normal subjects $[19,47]$, confirming the generality of this Weber's law-based property of temporal production. Pearson correlation coefficients $(r)$ between cognitive clock variance and period for H.J., control 1, and control 2 were $0.978,0.988$ and 0.979 respectively. These highlight the strength of the linear relationship, given that all correlations fell above 0.9 .

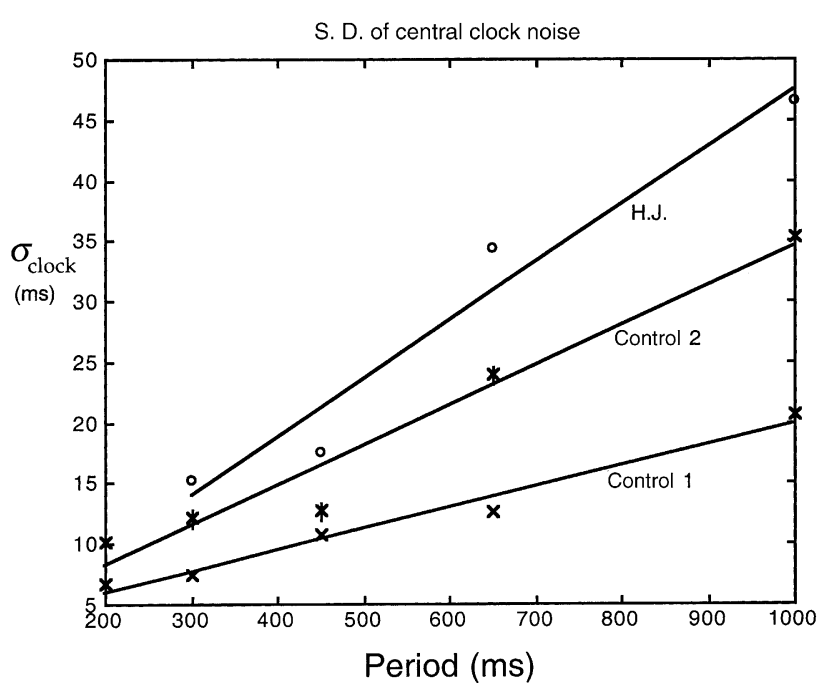

Fig. 3. Cognitive clock component of the extended Wing and Kristofferson model [58]. Values represent the standard deviation of central clock noise for each tapping speed averaged across the left and right hands. Data are shown separately for H.J. and the two control subjects. 
Table 3

Motor variance values $\left(\mathrm{ms}^{2}\right)$ and fraction of runs showing a clear AR1 structure for H.J. and the control subjects

\begin{tabular}{|c|c|c|c|c|c|c|}
\hline \multirow[t]{2}{*}{ Condition } & \multicolumn{6}{|l|}{ Subjects } \\
\hline & H.J. & Control 1 & Control 2 & H.J. & Control 1 & Control 2 \\
\hline LH (200 ms) & $\mathrm{a}$ & $7.82( \pm 9.7)$ & $33.52( \pm 66.3)$ & $\mathrm{a}$ & $4 / 4$ & $7 / 7$ \\
\hline RH (200 ms) & $\mathrm{a}$ & $-7.33( \pm 7.9)$ & $13.26( \pm 18.8)$ & a & $4 / 4$ & $5 / 5$ \\
\hline LH (300 ms) & $24.66( \pm 33.3)$ & $10.72( \pm 6.2)$ & $21.59( \pm 9.8)$ & $5 / 7$ & $4 / 4$ & $4 / 4$ \\
\hline RH (300 ms) & $21.64( \pm 25.2)$ & $7.49( \pm 14.9)$ & $-24.77( \pm 37.7)$ & $3 / 7$ & $3 / 4$ & $4 / 4$ \\
\hline LH (450 ms) & $39.22( \pm 21.3)$ & $13.49( \pm 23.9)$ & $69.64( \pm 35.2)$ & $4 / 4$ & $4 / 4$ & $4 / 4$ \\
\hline RH (450 ms) & $32.10( \pm 24.6)$ & $6.90( \pm 6.0)$ & $32.85( \pm 70.2)$ & $4 / 4$ & $4 / 4$ & $4 / 4$ \\
\hline LH (650 ms) & $45.56( \pm 128.8)$ & $25.84( \pm 22.3)$ & $60.27( \pm 93.7)$ & $3 / 5$ & $4 / 4$ & $3 / 4$ \\
\hline RH (650 ms) & $104.60( \pm 53.2)$ & $35.73( \pm 3.6)$ & $186.01( \pm 162.6)^{b}$ & $0 / 4$ & $1 / 4$ & $2 / 3^{\mathrm{b}}$ \\
\hline LH (1000 ms) & $360.60( \pm 320.9)$ & $58.93( \pm 93.2)^{\mathrm{c}}$ & $33.25( \pm 94.3)$ & $1 / 4$ & $3 / 7^{\mathrm{c}}$ & $3 / 4$ \\
\hline RH (1000 ms) & $797.00( \pm 531.3)$ & $27.62( \pm 37.0)$ & $111.1( \pm 382.5)$ & $4 / 4$ & $4 / 4$ & $3 / 4$ \\
\hline
\end{tabular}

${ }^{\mathrm{a}}$ H.J. was unable to perform this condition.

${ }^{\mathrm{b}}$ One outlier run was excluded when calculating this value.

${ }^{\mathrm{c}}$ This value was calculated from combined data taken from seven shorter tapping runs.

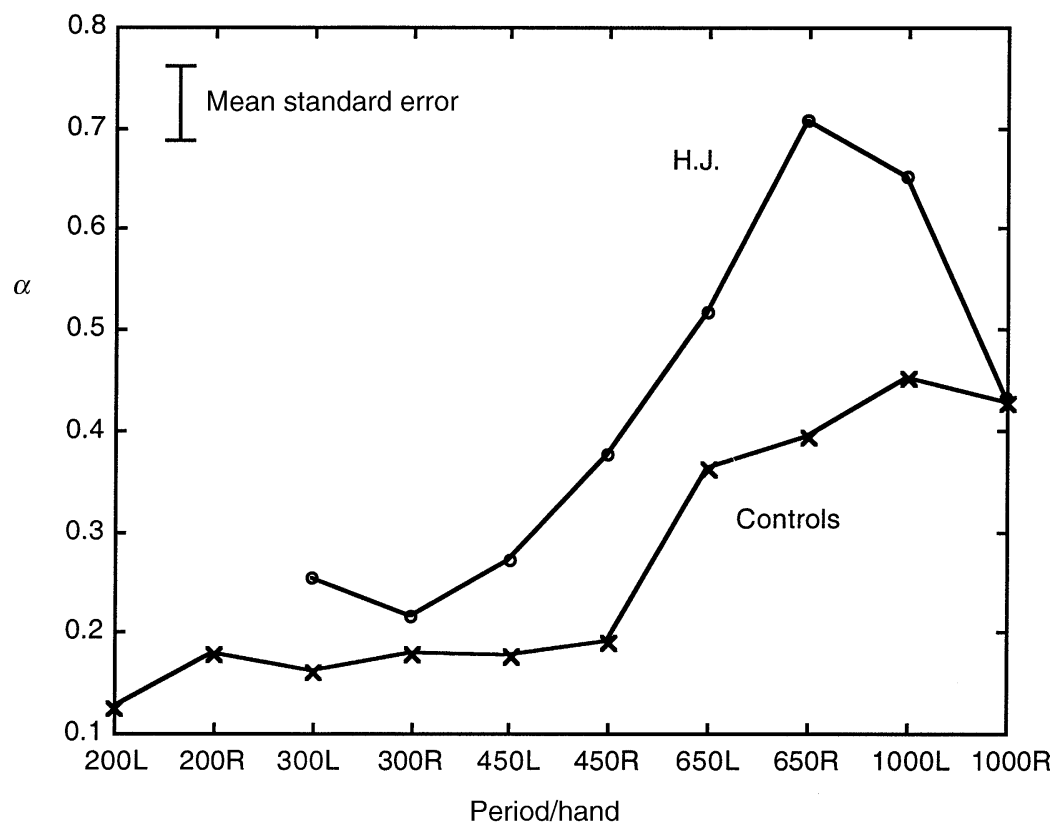

Fig. 4. Error-correction parameter of the Wing and Kristofferson model extended by Vorberg and Wing [58]. The values of $\alpha$ are shown for each tapping speed in the left and right hands for H.J. and the mean performance of the control subjects.

\subsubsection{Motor delay $\left(D_{n}\right)$}

In general, H.J.'s motor variance did not differ significantly from the control subjects across the conditions of the rhythmic tapping task (binomial sign test, $P=0.15$ ). Examination of the performance of each subject showed that control 1 exhibited the standard behaviour of motor variance, invariant as a function of speed (Table 3). ${ }^{1}$ Control 2 showed a possible modest increase in motor variance with speed, whilst H.J. showed a dramatic increase for the slowest speed $(1000 \mathrm{~ms})$. The performance of H.J. (and to a limited

\footnotetext{
${ }^{1}$ Motor delay variances are computed in the Wing and Kristofferson approach as a small difference of two large terms. Hence, they typically exhibit much greater variability than that of the central clock variances.
}

extent control 2) is suggestive of a possible breakdown of the independence of the motor (peripheral) and the clock (central) components of the rhythmic timing model at longer periods. Alternatively, the automaticity of the motor delay process may have been compromised by interruptions, such as attentional inconsistencies. This effect is likely attributable to age, compounded by neurological damage in the case of H.J.

\subsubsection{Error-correction mechanism $(\alpha)$}

In contrast to H.J.'s severely disturbed cognitive clock and his relatively normal motor processing, he showed greater values of the error-correction parameter $(\alpha)$ indicating superior error-correction processing (Fig. 4). In comparison to 
the control subjects, H.J.'s error-correction was significantly faster and more powerful for the conditions of the tapping task, despite having a more limited tempo range (binomial sign test, $P=0.004)$. This suggests that the control subjects may not have fully matched H.J.'s premorbid level of musical expertise. In any case, it would appear that H.J.'s error-correction process was unaffected by his lesion, and that this process can be separated from the clock and motor processes. Pressing $[47,48]$ has suggested that alpha should be roughly linear with period for speeds below about $800 \mathrm{~ms}$. The present data are consistent with this suggestion with correlations again greater than 0.9 for H.J. (0.977), control 1 (0.960), and control 2 (0.919).

\subsubsection{AR1 versus $A R 2$}

The tapping data generated by the control subjects consistently showed no significant improvement of fit for the AR2 model over the AR1 model (Table 3). In other words, the subjects were employing only first order error-correction, so that only the last error was used to adjust current tapping behaviour. ${ }^{2}$ H.J.'s performance appeared more erratic, both within and between tapping runs (Table 3). At the most comfortable tempo range $(450 \mathrm{~ms})$, he showed consistent use of the AR1 model. At other tempi, however, his tapping control was less consistent, sometimes showing second order error-correction and occasionally not conforming well to either model. General fatigue and reduced concentration may have contributed to his variable performance.

\section{Discussion}

In this paper, we have introduced into the clinical setting a recent model for deconstructing motor timing skill into three components. Specifically, synchronisation to an external source requires cognitive pulse generation, motor implementation, and error-correction. In comparing H.J. to two control subjects, we have found that both error-correction and cognitive clock standard deviations increased approximately linearly with period, in accordance with earlier results and theoretical predictions $[19,47]$.

Relative to the controls, H.J.'s error-correction capacity appeared unimpaired, while his cognitive clock process was erratic (exhibiting high variance). His peripheral motor process appeared relatively normal until pulse slowed down to about $1000 \mathrm{~ms}$, where motor variance rose dramatically. None of the subjects showed clear cut effects of hand, despite H.J.'s right-sided lesion.

Relative to the earlier work of Pressing and Jolley-Rogers [49], our first control subject acted like an expert while our second control was similar to their non-expert, perhaps reflecting the variable effects of age. In terms of his error-correction H.J. is also an expert, but he has deficiencies in other skill areas. In addition to the controls of this study,

\footnotetext{
${ }^{2}$ In mathematical terms, this is called a Markoff process.
}

H.J.'s performance was notably worse than a third older expert available from Pressing and Jolley-Roger's work [49] who closely matched the performance of our first control subject.

The main source of H.J.'s control problem lies in his production of reference clock pulses. Although he can still perceive and attempt to quench errors in his performance with a high skill level, he cannot control his output (clock pulses) with low enough variability to appear skillful. This seems to emphasise that error-correction addresses the output or the result of the central clock process and can maintain its role despite malfunction of pulse production. This dissociation between error and clock variance has also been recently found in patients with Parkinson's disease or cerebellar damage by Ivry and Pressing (journal submission).

In other words, modelling H.J.'s performance of the tapping task revealed that one component of this model was selectively disturbed following a right temporoparietal infarct. H.J. was unable to adequately generate regular clock intervals through a central cognitive mechanism. The bilateral nature of this disturbance precludes the possibility that impoverished sensory feedback of H.J.'s left upper limb fully accounted for the rhythmic timing deficit. The disturbance was considered to underlie all of H.J.'s rhythmic difficulties, including the inability to keep time to music (dancing, tapping, playing) or to spontaneously generate a steady pulse, and the inability to benefit from metric organisation when discriminating or reproducing novel rhythmic patterns. H.J.'s impaired bi-manual co-ordination whilst playing was also considered to partly reflect his rhythmic timing deficit [61].

In contrast to these difficulties, H.J.'s ability to discriminate between non-metrical rhythms, or to correctly classify non-metrical and metrical patterns did not differ significantly from the NC group. This dissociation in H.J.'s rhythmic performance likely reflects the different components of rhythmic function assessed by metrical and non-metrical tasks. In particular, metrical rhythmic patterns are hierarchically organised, with pulse typically comprising a low level of the hierarchical structure ([45,14,21]; see [6] for a review). Discrimination of metrical patterns is ratio based, requiring the listener to perceive two or more adjacent temporal levels that are nested within each other. Discrimination of non-metrical patterns does not require the listener to perceive a hierarchical temporal structure. It can occur solely on the basis of the estimation of note duration [10]. Presumably the classification of regularity or non-regularity in a temporal sequence (for example, metrical classification) can be performed using either strategy, with differing contexts favouring one method over the other (for example, see [46]).

Thus, H.J.'s findings supported a distinction between impaired pulse generation and metrical pattern discrimination, and preserved duration estimation. These findings, in conjunction with the results of previous research, highlight some of the essential components of the cognitive architecture of rhythmic function, which we have summarised in Table 4. 
Table 4

Some of the essential components of rhythmic function

\begin{tabular}{|c|c|c|}
\hline Processing level & Psychological components & Possible neural correlates \\
\hline \multirow[t]{4}{*}{$\begin{array}{l}\text { I Input, perceptual, and } \\
\text { cognitive processing }\end{array}$} & $\begin{array}{l}\text { Record tone onsets/detect } \\
\text { events (e.g. [15]) }\end{array}$ & $\begin{array}{l}\text { Bilateral temporal cortices } \\
\text { (e.g. [36]) }\end{array}$ \\
\hline & Estimate time intervals (e.g. $[10,9,33])$ & $\begin{array}{l}\text { Bilateral temporal cortices (e.g. [36]) or Left temporal } \\
\text { cortex preferentially (e.g. }[33,46] \text { ) }\end{array}$ \\
\hline & Infer a pulse (e.g. $[45,8,21,35])$ & Right temporal cortex (e.g. [11]) \\
\hline & Infer meter (e.g. $[27,45,26,24,14,21,23,35,55])$ & $\begin{array}{l}\text { Bilateral temporal cortices (e.g. [25]) or Right tem- } \\
\text { poroparietal cortex (e.g. }[37,44] \text { ) }\end{array}$ \\
\hline \multirow{3}{*}{$\begin{array}{l}\text { II Building/preparing } \\
\text { an action }\end{array}$} & Update short-term timing memory (e.g. $[10,15])$ & Right temporal cortex (e.g. [36]) \\
\hline & $\begin{array}{l}\text { Tune clock pulse; set mean and restrict variance as far } \\
\text { as conditions allow (e.g. }[68,69,58,49])\end{array}$ & Right temporoparietal cortex (e.g. H.J.) \\
\hline & $\begin{array}{l}\text { Use asynchronies to compute error-corrections to be } \\
\text { applied (e.g. }[58,49])\end{array}$ & $\begin{array}{l}\text { Distributed (e.g. the motor system, including subeortical } \\
\text { structures; [56,32]; for a more general reference see } \\
\text { [22]) }\end{array}$ \\
\hline \multirow[t]{2}{*}{ III Motor action } & $\begin{array}{l}\text { Use error corrected clock pulse values to trigger motor } \\
\text { process (e.g. }[58,49])\end{array}$ & $\begin{array}{l}\text { Distributed (e.g. the motor system, including subeortical } \\
\text { structures; [56,32]; for a more general reference see } \\
\text { [22]) }\end{array}$ \\
\hline & $\begin{array}{l}\text { Monitor tempo invariance over longer time spans to } \\
\text { maintain stationarity of clock process parameters }\end{array}$ & $\begin{array}{l}\text { Right temporoparietal cortex plus other areas may be } \\
\text { involved (e.g. H.J.) }\end{array}$ \\
\hline
\end{tabular}

Note: These processes are overlapping and do not follow in strict temporal order. Furthermore, column 2 is an elaboration of column 1.

In its current form, this summary primarily relates to 'keeping the beat' in music, but may be more broadly applicable to other skilled motor behaviours.

As shown in Table 4, one of the basic functions of the rhythmic system is to infer and construct a regular pulse. This is a cognitive mechanism that appears to rely on the integrity of the right temporal lobe. H.J. and the case reported by Fries and Swihart [11] suggest that the critical lesion involved right temporal neocortex rather than subcortical structures (i.e. the basal ganglia). McFarland and Fortin [31] also reported a case of amusia associated with a right temporoparietal infarct. This case appeared to present with similar amusic features to H.J., however, pulse generation was not formally assessed. There are no known cases of impaired pulse generation following damage to the left hemisphere, although in general, the number of cases in which this has been examined is small, indicating that further research is required to investigate the specificity of the lesion.

The ability to estimate time intervals and to infer meter also represent essential components of the rhythmic system. Neuropsychologically, these functions have shown more variable lateralisation effects (Table 4). As stated by Penhune and co-workers [36]:

"review of the neuropsychological literature related to both basic temporal parameters and musical rhythm links these functions to the auditory cortices of the temporal lobe, but does not demonstrate a more precise localisation within auditory cortex, nor any consistent lateralisation to either the left or right hemisphere." (p. 317)

Reasons for the variability of these effects may be partly task related, given the numerous levels on which rhythmic stimuli can be processed. An exception, however, appears to be the processing of short-term timing memory (Table 4), which has been ascribed to the functioning of the right anterior secondary auditory cortex [36]. This is in keeping with previous research by Zatorre and co-workers [70,71] implicating the role of the right temporal lobe in working memory for pitch.

Of particular interest in this study, H.J. showed impaired ability to monitor the tempo of his performance over longer time spans (i.e. $\geq 10 \mathrm{~s}$ ). This function has received considerably less attention in the research literature. For example, it was not included in the modelling approach of Wing and Kristofferson as they used rather short tapping runs (approximately 30 taps). Over longer runs, the ability to maintain tempo invariance has been assumed to occur automatically and thus, to produce stationarity within the data. Stationarity typically reflects uniform values of control parameters over the course of a run, such as the mean value of the cognitive clock. For longer runs, however, this parameter maintenance process must be considered to be a potentially significant source of error, particularly in individuals with potential control deficiencies. This appears likely in the case of H.J., as reflected in his erratic use of AR1 versus AR2 models both within and between tapping runs, in comparison to the normal controls.

It is possible that the mechanism for clinical inflation of variance is non-stationarity. In behavioural terms, this would be expressed as an inability to maintain control structures, such as tempo, over extended periods of time. This was clearly reflected in H.J.'s instrumental performance of large-scale works, where the tempo of the main theme of the work would vary noticeably throughout the piece. In contrast, H.J.'s ability to reproduce shorter, familiar rhythms appeared intact, presumably because performance of these rhythms did not invoke this control mechanism. To the authors' knowledge, H.J. is the first neuropsycholog- 
ical case report of a deficit of this nature. His difficulties implicate the role of the right temporoparietal cortex in maintaining tempo invariance, but other regions may also be involved (Table 4).

Finally, in accordance with the extended Wing and Kristofferson model $[49,58,68,69]$, the cognitive architecture of rhythmic function must incorporate motor output and error-correction processes. These processes are considered to have distributed cortical and subcortical representation, including premotor cortex, supplementary motor area (corticospinal motor expression), motor cortex, basal ganglia and cerebellum $[22,32,56]$.

\section{Conclusion}

Use of the autoregressive extended Wing and Kristofferson model of rhythmic timing provided an appropriate means with which to characterise the nature of H.J.'s arrhythmia. In particular, his impaired ability to generate steady clock pulses appeared to form the underlying basis of a global rhythmic disturbance. This was clearly dissociated from preserved motor and error-correction processes, as quantified by the modelling approach of Wing, Kristofferson, Vorberg and Pressing. This method of analysis provoked discussion of the essential components of the cognitive architecture of rhythmic function with respect to their cerebral lateralisation and localisation. Overall, the findings suggested that the functioning of the right temporal auditory cortex may be fundamental to synchronisation or 'keeping the beat' in music. The tapping task has high face validity, given the importance of 'keeping the beat' when performing with other musicians. The approach provides, therefore, a new paradigm for future neuropsychological research examining rhythmic disturbances.

\section{Acknowledgements}

The authors would like to express their gratitude to H.J. and the control subjects for participation in this study. Recognition of the important contribution of others is also warranted, including Associate Professor Michael Saling for assistance with the interpretation of H.J.'s CT scan, Young Ho Kim for writing the recording and data analysis software used in the isochronous tapping task, Gary Jolley-Rogers for running the data analysis of the tapping task, and Dr Robert Zatorre for permission to use his auditory memory tasks. This research was partly supported by an ARC Grant A79533005.

\section{References}

[1] Bentley A, Measures of musical abilities. Great Britain: Nfer-Nelson, 1985.
[2] Benton AL, The amusias. In: Critchley M, Henson RA, editors. Music and the brain: studies in the neurology of music. London: William Heinemann Medical Books, 1980. pp. 378-97.

[3] Berman IW. Musical functioning, speech lateralisation and the amusias. South African Medical Journal 1981;17:78-81.

[4] Brust JCM. Music and language: musical alexia and agraphia. Brain 1980;103:367-92.

[5] Chase CI. Elementary Statistical Procedures, 3rd edition. Singapore: McGraw-Hill, 1985.

[6] Clarke, E.F., Rhythm and timing in music. In: Deutsch D, editors. The psychology of music, 2nd ed. New York: Academic Press, 1999. pp. $473-500$.

[7] Denckla MB. Development of motor co-ordination in normal children. Developmental Medicine and Child Neurology 1974;16:729-41.

[8] Dowling WJ, Harwood DL, Music cognition. New York: Academic Press, 1986.

[9] Flückiger M, The effects of time pressure on duration discrimination. In: Michon JA, Jackson JL, editors. Time, mind, and behaviour. Berlin: Springer-Verlag, 1985. pp. 131-9.

[10] Fraisse P, Rhythm and tempo. In: Deutsch D, editor, The psychology of music. New York: Academic Press, 1982: 149-80.

[11] Fries W, Swihart AA. Disturbance of rhythm sense following right hemisphere damage. Neuropsychologia 1990;28:1317-23.

[12] Gordon HW. Left hemisphere dominance for rhythmic elements in dichotically-presented melodies. Cortex 1978;14:58-70.

[13] Gordon HW, Bogen JE. Hemispheric lateralization of singing after intracarotid sodium amylobarbitone. Journal of Neurology, Neurosurgery, and Psychiatry 1974;37:727-38.

[14] Handel S. Using polyrhythms to study rhythm. Music Perception 1984;1:465-84

[15] Handel S. Listening. Cambridge: The MIT Press, 1989.

[16] Hary D, Moore GP. On the performance and stability of human metronome-synchronization strategies. British Journal of Mathematical and Statistical Psychology 1987;40:109-24.

[17] Henson RA. Amusia. In: Frederiks JAM, editor. Handbook of clinical neurology, vol. 1. New York: Elsevier, 1985. pp.483-90.

[18] Ibbotson N, Morton J. Rhythm and dominance. Cognition 1981;9:125-38.

[19] Ivry RB, Hazeltine RE. The perception and production of temporal intervals across a range of durations: evidence for a common timing mechanism, human perception and performance. Journal of Experimental Psychology 1995;21:1-12.

[20] Ivry RB, Pressing J. Temporal error-correction in Parkinson's and cerebellar patients, in preparation.

[21] Johnson-Laird PN. Rhythm and meter: a theory at the computational level. Psychomusicology 1991;10:88-106.

[22] Kandel ER, Schwartz JH, Jessell TM, editors. Principles of neural science, 4th ed. Connecticut: Appleton \& Lange, 2000.

[23] Lee CS, The perception of metrical structure: experimental evidence and a model. In: Howell P, West R, Cross I, editors. Representing musical structure. London: Academic Press, 1991. pp. 59-127.

[24] Lerdahl F, Jackendoff R. A generative theory of tonal music. Cambridge: The MIT Press, 1983.

[25] Liégeois-Chauvel C, Peretz I, Babaï M, Laguitton V, Chauvel P. Contribution of different cortical areas in the temporal lobes to music processing. Brain 1998;121:1853-67.

[26] Longuet-Higgins HC, Lee CS. The perception of musical rhythms. Perception 1982;11:115-28.

[27] Martin JG. Rhythmic (hierarchical) versus serial structure in speech and other behaviour. Psychological Review 1972;79:487-509.

[28] Mates J. A model of synchronisation of motor acts to a stimulus sequence, Part I. Biological Cybernetics 1994a;70:463-73.

[29] Mates J. A model of synchronisation of motor acts to a stimulus sequence, Part II. Biological Cybernetics 1994;70:475-84.

[30] Mavlov L. Amusia due to rhythm agnosia in a musician with left hemisphere damage: a non-auditory supramodal defect. Cortex 1980;16:331-8. 
[31] McFarland HR, Fortin D. Amusia due to right temporoparietal infarct. Archives of Neurology 1982;39:725-7.

[32] Miall RC, Imamizu H, Miyauchi S. Activation of the cerebellum in co-ordinated eye and hand tracking movements: an fMRI study. Experimental Brain Research 2000;135:22-33.

[33] Nicholls MER. Temporal processing asymmetries between the cerebral hemispheres: evidence and implications. Laterality 1996;1:99-137.

[34] Nicholls MER, Schier M, Stough CKK, Box A. Psychophysical and electrophysiologic support for a left hemisphere temporal processing advantage. Neuropsychiatry, Neuropsychology, and Behavioural Neurology 1999;12:11-6.

[35] Parncutt R. A perceptual model of pulse salience and metrical accent in musical rhythms. Music Perception 1994;11:409-64.

[36] Penhune VB, Zatorre RJ, Feindel WH. The role of auditory cortex in retention of rhythmic patterns as studied in patients with temporal lobe removals including Heschl's gyrus. Neuropsychologia 1999;37:315-31.

[37] Peretz I. Processing of local and global musical information by unilateral brain-damaged patients. Brain 1990;113:1185-205.

[38] Peretz I. Auditory atonalia for melodies. Cognitive Neuropsychology 1993a;10:21-56.

[39] Peretz I. Auditory agnosia: a functional analysis. In: McAdams S, Bigand E, editors. Thinking in sound: the cognitive psychology of human audition. Oxford: Clarendon Press, 1993b. pp. 199-230.

[40] Peretz I, Kolinsky R. Boundaries of separability between melody and rhythm in music discrimination: a neuropsychological perspective. The Quarterly Journal of Experimental Psychology 1993;46:301-25.

[41] Peretz I, Kolinsky R, Tramo M, Labrecque R, Hublet C, Demeurisse $\mathrm{G}$, et al. Functional dissociations following bilateral lesions of auditory cortex. Brain 1994;117:1283-301.

[42] Peretz I, Morais J. Music and modularity. Contemporary Music Review 1989;4:279-93.

[43] Peretz I, Morais J. Specificity for music. In: Boller F, Grafman J, editors. Handbook of neuropsychology, vol. 8. Amsterdam: Elsevier, 1993. pp. 373-90.

[44] Polk M, Kertesz A. Music and language in degenerative disease of the brain. Brain and Cognition 1993;22:98-117.

[45] Povel DJ. Internal representation of simple temporal patterns: human perception and performance. Journal of experimental psychology 1981;7:3-18

[46] Platel H, Price C, Baron JC, Wise R, Lambert J, Frackowiak RSJ, et al. The structural components of music perception: a functional anatomical study. Brain 1997;120:229-43.

[47] Pressing J. The referential dynamics of cognition and action. Psychological Review 1999;106:714-47.

[48] Pressing J. Error-correction processes in temporal pattern production. Journal of Mathematical Psychology 1998;42:63-101.

[49] Pressing J, Jolley-Rogers G. Spectral properties of human cognition and skill. Biological Cybernetics 1997;76:339-47.

[50] Pressing J, Summers J, Magill J. Cognitive multiplicity in polyrhythmic performance: human perception and performance. Journal of experimental psychology 1996;22:1127-48.

[51] Robin DA, Tranel D, Damásio H. Auditory perception of temporal and spectral events in patients with focal left and right cerebral lesions. Brain and Language 1990;39:539-55.

[52] Robinson GM, Solomon DJ. Rhythm is processed by the speech hemisphere. Journal of Experimental Psychology 1974;102:508-11.
[53] Schulze H-H. The error-correction model for the tracking of a random metronome: statistical properties and an empirical test. In: Macar F, Pouthas V, Friedman WJ, editors. Time, action, and cognition. Dordrecht: Kluwer, 1992. pp. 275-86.

[54] Swisher L, Hirsh IJ. Brain damage and the ordering of two temporally successive stimuli. Neuropsychologia 1972;10: $137-52$.

[55] Todd NPMcAngus. The auditory primal sketch: a multi-scale model of rhythmic grouping. Journal of New Music Research 1994;23: 25-70.

[56] Todd NPMcAngus. O'Boyle DJ, Lee CS. A sensory-motor theory of rhythm, time perception and beat induction. Journal of New Music Research 1999;28: 5-29.

[57] Turvey MT, Schmidt RC, Rosenblum LD. Clock and motor components in absolute coordination of rhythmic movements. Neuroscience 1989;33:1-10.

[58] Vorberg D, Wing A. Modelling variability and dependence in timing. In: Heuer H, Keele SW, editors. Handbook of perception and action, vol. 2. London: Academic Press, 1996. pp. 181-262.

[59] Wechsler, D. Wechsler adult intelligence scale-revised [manual]. New York: Psychological Corporation, 1981.

[60] Wilson, SJ. An exploration of the cerebral lateralisation of musical function. Doctoral Dissertation, The University of Melbourne, Australia, 1996.

[61] Wilson SJ, Pressing J. Neuropsychological assessment and modelling of musical deficits. In: Pratt RR, Erdonmez Grocke D, editors. Music medicine, vol. 3. Music medicine and music therapy: expanding horizons. Melbourne: The University of Melbourne, 1999. pp. $47-74$.

[62] Wilson SJ, Pressing J, Wales RJ, Pattison P. Cognitive models of music psychology and the lateralisation of musical function within the brain. Australian Journal of Psychology 1999;51:125-39.

[63] Wilson SJ, Wales RJ. An exploration of children's musical compositions. Journal of Research in Music Education 1995;43:94 111.

[64] Wilson SJ, Wales RJ, Pattison P. The representation of tonality and meter in children aged 7 and 9. Journal of Experimental Child Psychology 1997;64:42-66.

[65] Wing AM. Effects of type of movement on the temporal precision of response sequences. British Journal of Mathematical and Statistical Psychology 1977;30:60-72.

[66] Wing AM, The long and short of timing in response sequences. In: Stelmach GE, Réquin J, editos. Tutorials in motor behaviour. Amsterdam: North Holland, 1980. pp. 469-86.

[67] Wing AM, Church RM, Gentner DR. Variability in the timing of responses during repetitive tapping with alternate hands. Psychological Research 1987;51:28-37.

[68] Wing AM, Kristofferson AB. The timing of interresponse intervals. Perception and Psychophysics 1973a;13:455-60.

[69] Wing AM, Kristofferson AB. Response delays and the timing of discrete motor responses. Perception and Psychophysics 1973;14:512.

[70] Zatorre RJ, Evans AC, Meyer E. Neural mechanisms underlying melodic perception and memory for pitch. The Journal of Neuroscience 1994;14:1908-19.

[71] Zatorre RJ, Samson S. Role of the right temporal neocortex in retention of pitch in auditory short-term memory. Brain 1991;114:2403-17. 